\title{
Down-regulation of miR-193b-3p and miR-376a-3p in Chronic Lymphocytic Leukemia
}

\author{
Faezeh Namazi ${ }^{1}$, Nasrin Hadi ${ }^{1}$, Mansour Moghimi², Amir Eshaghiyan ${ }^{3}$, and \\ Behnaz Nateghi4 \\ ${ }^{1}$ Medical Biotechnology Research Center, Ashkezar Branch, Islamic Azad University, Ashkezar, \\ Yazd, Iran \\ ${ }^{2}$ Department of Pathology, Shahid Sadoughi University of Medical Sciences, Yazd, Iran \\ ${ }^{3}$ Department of Genetics, Arsanjan Branch, Islamic Azad University, Arsanjan, Shiraz, Iran \\ ${ }^{4}$ Department of Biochemistry, Faculty of Science, Nourdanesh Institutions of Higher Education, \\ Meimeh, Isfahan, Iran
}

\section{Abstract}

Background: Chronic lymphocytic leukemia (CLL) is the most common adult human leukemia. Studies revealed that microRNAs (miRNAs) can function as oncogenes or tumor suppressors in CLL and that the expression of miRNAs, such as miR-193b-3p and miR-376a-3p change in several diseases. We aimed to elucidate the changes in miR193b-3p and miR-376a-3p expression in CLL and determine their potential as diagnostic biomarkers for this disease.

Corresponding Author: Behnaz Nateghi; email:

behnaz.nateqi@gmail.com

Production and Hosting by Knowledge E

(c) Faezeh Namazi et al. This article is distributed under the terms of the Creative

Commons Attribution License, which permits unrestricted use and redistribution provided that the original author and source are credited.

Editor-in-Chief:

Dr. Alireza Rafiei
Materials and Methods: We investigated miR-193b-3p and miR-376a-3p expression by quantitative real-time PCR in peripheral blood mononuclear cells of 30 patients with CLL and 30 healthy individuals. Moreover, in silico molecular enrichment analysis was conducted on predicted and validated targets of miR-193b-3p and miR-376a-3p from the miRecords and miRTarBase databases.

Results: The expression of miR-193b-3p and miR-376a-3p was significantly different between the two groups $(\mathrm{P}<0.0001$ and $\mathrm{P}<0.0001$, respectively).

Conclusion: Based on these findings, miR-193b-3p and miR-376a-3p could be novel biomarkers for the early diagnosis of CLL and could be used to design new CLL control strategies.

Keywords: chronic lymphocytic leukemia, miR-193b-3p, miR-376a-3p, miRNA

\section{G OPEN ACCESS}




\section{Introduction}

Chronic lymphocytic leukemia (CLL) is a clonal proliferative tumor of mature $B$ lymphocytes characterized by the lymphocyte accumulation in the peripheral blood, bone marrow, and lymph nodes [1]. CLL is characterized by asymmetrical proliferation and apoptosis of leukemia cells along with expressing the CD5 and CD19 markers. Several chromosomal abnormalities were detected as the most common abnormalities in CLL, including deletions at 13q, 11q, 17p; and trisomy 12 [2, 3]. This leukemia is the most common type of leukemia in the western world, causing $~ 5,000$ mortalities in the United States annually [4]. Most patients with CLL are able to survive for several years and show mild symptoms [5]. One of the important factors to be considered in cancer therapy, which helps to plan and choose better treatment options, is the early diagnosis. Discovery of biomarkers at different levels, including genomics, transcriptomics, and proteomics levels can help to better diagnose and treat several cancers, including CLL [6]. Unfortunately, the precise molecular mechanisms underlying the CLL progress still unclear, which limit early diagnosis and timely treatment. Thus, tracking the molecular mechanisms of CLL progression and its biomarkers to improve the likelihood of early diagnosis have become the focus of CLL research. Some evidence suggests that multiple genes, miRNAs, and cellular pathways are involved in the incidence and progression of CLL $[7,8]$. MicroRNAs (miRNAs) are short non-coding regulatory RNAs that control the gene expression at the post-transcriptional level by binding to the $3^{\prime}$-untranslated region (UTR) or 5'UTR of their mRNA targets, resulting in inhibition or degradation of mRNA. miRNAs play a significant role in cancer progression by acting either as oncogenes or tumor suppressors [9]. miRNAs expression profiles have already entered cancer clinics as diagnostic biomarkers to recognize tumor initiation, progression, and response to treatment in patients with cancer [10]. The first human disease-related miRNAs, miR-15 and miR-16 at $13 q 14$, were characterized in CLL [11, 12]. Some evidences indicated that deregulation of miRNAs could be associated with CLL [13]. Moreover, some studies have shown that the expression of miR-193b-3p is altered in several biological samples of cancer patients, such as acute lymphoblastic leukemia (ALL), prostate cancer, and ovarian cancer [1416]. Additionally, changes in the expression of $m i R-376 a-3 p$ have been associated with various human diseases, such as ovarian cancer and hepatocellular tumor of bone [1517]. However, the roles of miR-193b-3p and miR-376a-3p in CLL disease has remained unexplored, till date. In this study, the expression of miR-193b-3p and miR-376a-3p was examined by quantitative real-time PCR in peripheral blood mononuclear cells (PBMCs) of patients with CLL and healthy individuals. Therefore, the aim of our study was to 
determine whether the deregulated expression of $m i R-193 b-3 p$ and miR-376a-3p (as these two miRNAs are involved in CLL) can be used as a new diagnostic biomarker in patients with CLL.

\section{Materials and Methods}

\subsection{Patients and controls}

Blood samples were collected from patients with CLL and from randomly selected healthy individuals (both male and female). Totally, 60 samples, including 30 from patients with CLL [diagnosed in the Omid Hospital (Isfahan, Iran)] and 30 from healthy individuals, were selected for the study. CLL was diagnosed based on blood cell count and cell morphology. The exclusion criteria were as follows: (i) CLL diagnosis more than 12 months before registration; (ii) Clinical Binet stage B or stage C; (iii) need for therapy according to National Cancer Institute $(\mathrm{NCl})$ guidelines [19]. Six milliliters of peripheral blood was collected in EDTA-containing tubes and immediately transported on ice to the laboratory. The Ethics Committee of the Omid Hospital (Isfahan, Iran) approved the protocol of this study. Written informed consent was obtained from all participating individuals prior to sample collection.

\subsection{Complete blood count}

Complete blood count (CBC) test was assessed using CA\&XN-Series TM Automated Hematology Analyses (Kobe, Japan). The Sysmex XN series uses fluorescence flow cytometry. Some variables associated with CLL were determined by this device.

\subsection{Peripheral blood mononuclear cell isolation from blood}

Peripheral blood mononuclear cells (PBMCs) were isolated from blood samples by density gradient lymphoprep (Bio Sera, Kansas City, USA) based on the manufacturer's protocol. Mononuclear cells, monocytes, and lymphocytes have lower densities in comparison with erythrocytes and leukocytes; therefore, after centrifugation, they remain in an intermediate phase. Briefly, $4 \mathrm{ml}$ of blood was diluted at a ratio of 1:1 with physiological saline and gradually added to the $4 \mathrm{ml}$ lymphoprep solution gradient in a Falcon tube. The tubes were centrifuged at $800 \times g$ for $30 \mathrm{~min}$, and then PBMCs were transferred 
from the middle phase into $2-\mathrm{mL}$ RNAase-free microtubes and frozen at $-70{ }^{\circ} \mathrm{C}$ until next step.

\subsection{MiRNA extraction}

MiRNA was extracted from PBMCs using miRNA Hybrid-R (Geneall, Seoul, Korea) based on the manufacturer's instructions. Quality of the extracted miRNA was determined according to the 260/280 absorbance ratio, measured by NanoDrop spectrometer (Thermo Scientific, Waltham, MA, USA).

\subsection{Complementary DNA synthesis and real-time polymerase chain reaction}

Complementary DNA (cDNA) synthesis for miR-193b-3p and miR-376a-3p was carried out using a universal cDNA synthesis kit (Exiqon, Denmark) using poly-A tailing, according to manufacturer's instructions. Real-time quantitative PCR reactions were carried out in triplicate by using standard protocols with an ABI PRISM 7500 instrument (Applied Biosystems, USA). Briefly, in a total volume of $10 \mu \mathrm{l}, 20 \mathrm{ng} / \mathrm{\mu l}$ of cDNAs were added to a master mix comprising $10 \mathrm{pmol} / \mathrm{\mu l}$ of each miR-193b-3p or miR-376a-3p primers (Exeqon, Denmark) and $5 \mathrm{ml}$ of SYBR premix ExTaq II (TaKaRa, Kusatsu, Shiga Prefecture, Japan). The program for the run was set as follows: $95{ }^{\circ} \mathrm{C}$ for 15 min followed by 40 cycles of $95{ }^{\circ} \mathrm{C}$ for $15 \mathrm{~s}, 60{ }^{\circ} \mathrm{C}$ for $30 \mathrm{~s}$, and $72{ }^{\circ} \mathrm{C}$ for $30 \mathrm{~s}$. The PCR reaction was followed by a melting curve program $\left(60-75{ }^{\circ} \mathrm{C}\right.$ with a temperature transition rate of $1{ }^{\circ} \mathrm{C} \mathrm{s}^{-1}$ and a continuous fluorescence reading). Real-time PCR data analysis was performed using the $\triangle \triangle C T$ method ${ }^{20}$ and the final data were normalized using small nuclear RNA, U6, expression level as an endogenous control.

\subsection{Statistical analysis}

For the statistical study, Graph Pad Prism statistical software, version 5.01 (Graph Pad, San Diego, CA, USA) was used. Normality was evaluated by the Kolmogorov-Smirnov test. The independent samples $t$-test was applied to analyze the data between groups. For all the tests, $p \leq 0.05$ was considered to indicate significance. 


\subsection{Molecular enrichment analysis}

In order to find the appropriate miRNA for CLL, the miRWalk database was used. Moreover, to perform molecular enrichment analysis on miR-193b-3p and miR-376a-3p, several in silico online databases were used. Briefly, miRecords and miRTarBase databases were employed to find predicted and validated targets of miR-193b-3p and miR-376a-3p $[21,22]$.

\section{Results}

\subsection{Clinical and biological features of patients}

In the current study, 30 patients with CLL (mean age: $60.87 \pm 1.591$ years, range: $45-81$ years, 18 males and 12 females, mean of disease duration: $1 \pm 0.67$ years) and 30 healthy individuals (mean age: $44.03 \pm 1.952$ years, range: $25-68$ years, 21 males and 9 females) were studied. Patients and controls were sufficiently matched in terms of age and sex $(P=0.11$ and 0.99 , respectively). It was observed that, in the patients, the levels of some variables associated with CLL, including WBC, RBC, monocyte, lymphocyte, and platelet counts, were higher than the normal values. However, there was no significant difference between the RBC levels in the healthy individuals and patients (Table 1).

TABLE 1: Hematological parameters of patients with CLL.

\begin{tabular}{|c|c|c|c|}
\hline Characteristics & Control & CLL & P-value \\
\hline Number of subjects & 30 & 30 & - \\
\hline Mean number of WBCs & $7.077 \pm 0.3239$ & $63.87 \pm 16.94$ & 0.001 \\
\hline Range (cells per $\mathrm{mcL} / 10^{3}$ ) & 4.5-9.9 & $6.03-451$ & \\
\hline Mean number of RBCs & $4.564 \pm 0.1539$ & $4.531 \pm 0.1661$ & 0.8835 \\
\hline Range (cells per mcL/10 ${ }^{6}$ ) & $3.29-5.98$ & $1.6-5.7$ & \\
\hline Mean number of Monocytes & $0.4463 \pm 0.04840$ & $5.958 \pm 2.011$ & 0.0081 \\
\hline Range (cells per $\mathrm{mcL} / 10^{3}$ ) & $0.04-0.7$ & $0.43-45.19$ & \\
\hline Mean number of Lymph & $2.530 \pm 0.1377$ & $43.67 \pm 15.34$ & 0.009 \\
\hline Range (cells per $\mathrm{mcL} / 10^{3}$ ) & $1.2-3.7$ & $2.38-408.03$ & \\
\hline Mean number of PLTs & $302.3 \pm 17.85$ & $135.9 \pm 8.493$ & $<0.0001$ \\
\hline Range $\left(\mathrm{mcL} / 10^{3}\right)$ & $155-420$ & $21-273$ & \\
\hline
\end{tabular}




\section{2. $m i R-193 b-3 p$ expression}

The expression of miR-193b-3p was evaluated by quantitative real-time PCR in patients with CLL $(n=30)$ and healthy individuals $(n=30)$. The Ct values of real-time PCR were used for quantification of relative gene expression by the $2^{-\Delta \Delta C t}$ method. A significant reduction was observed in the expression of miR-193b-3p in patients with CLL compared to that in healthy individuals with a $\mathrm{P}<0.0001$ (Figure 1). Thus, our results suggest that miR-193b-3p may be useful as a novel biomarker for patients with CLL.

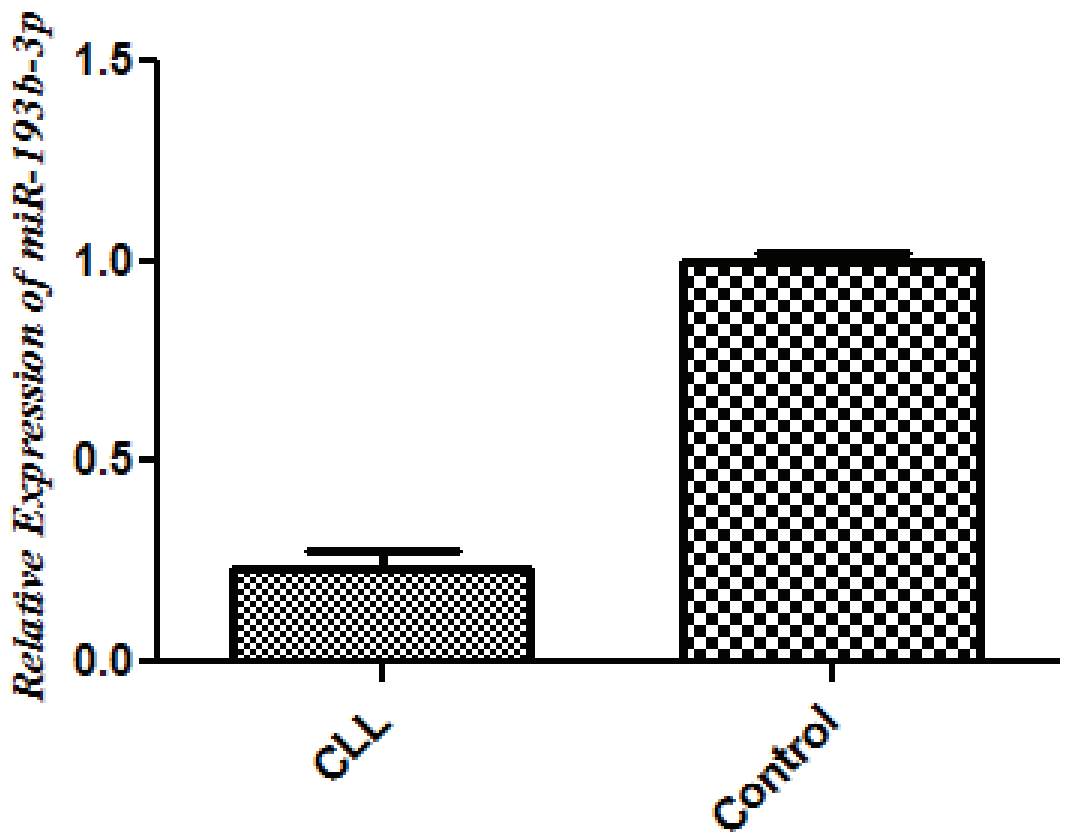

\section{Groups}

Figure 1: Relative expression of miR-193b-3p in patients with CLL and healthy individuals. Relative quantification for the miR-193b-3p was significantly different between CLL and control groups with a P < 0.0001. Error bars indicate SE.

\section{3. $m i R-376 a-3 p$ expression}

To explore the potential roles of $m i R-376 a-3 p$ in patients with CLL, we examined the expression level of miR-376a-3p in patients with CLL versus healthy individuals by qRTPCR. Our results indicated that the expression level of miR-376a-3p was significantly downregulated in the patients with CLL compared to that in healthy individuals $(\mathrm{P}<$ 0.0001) (Figure 2). These results show that miR-376a-3p was significantly decreased in the cancer patients and probably plays an important role in CLL. 


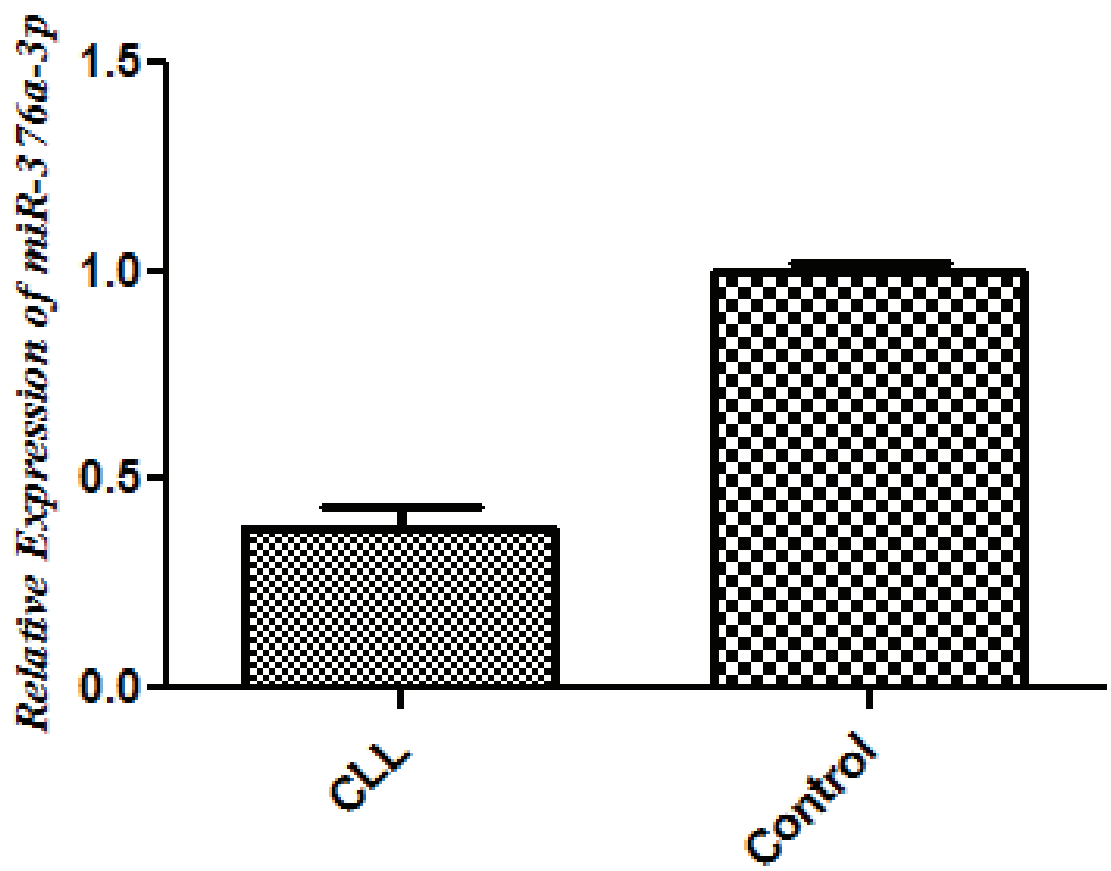

Groups

Figure 2: Relative expression of $m i R-376 a-3 p$ in patients with CLL and healthy individuals. Relative quantification for the miR-376a-3p was significantly different between CLL and control groups with a $\mathrm{P}<$ 0.0001. Error bars indicate SE.

\subsection{Molecular enrichment analysis}

Using the miRecords database, 76 and 34 mRNAs were identified as predicted targets of miR-193b-3p and miR-376a-3p, respectively (Supplementary Table $1 \& 2$ ). All predicted targets were confirmed by at least five prediction databases. Similarly, using the miRTarBase database, 9 and 5 mRNAs were obtained as validated targets of miR-193b-3p and miR-376a-3p, respectively. The validated targets of miR-193b-3p that were obtained from the miRTarBase database included CCND1, PLAU, MCL1, ETS1, MAX, KRAS, RAD51, $N F 1$, and SMAD3. Five validated targets were also identified for miR-376a-3p, including PIK3R1, IGF1R, PCna KLF15, and CASP8. All the validated targets in the miRTarBase database were supported by strong experimental evidences, including reporter assays, western blots, and quantitative real-time PCR.

\section{Discussion}

Previous studies have indicated that prognostic prediction in CLL is based on V-gene mutations (CD38 and ZAP-70) and various types of chromosomal deletions, such as 17q13, 6q21, 11q23, and 13q14 in CLL [23]. Despite the progress made in monitoring 
such patients, an accessible and reliable biomarker for diagnosis is still lacking. Early diagnosis of CLL would enable us to design more effective therapies. Circulating miRNAs could represent the available biomarkers for CLL [24]. miRNAs are well-known factors that affect the clinical phase of CLL. CLL was among the first human cancers discovered that was clearly related influenced by changes in miRNA expression. Deletion of $m i R$ $15 a$ and miR-16 in CLL was found to result in the overexpression of the BCL-2 gene [25]. In order to develop an miRNA-based biomarker for CLL or any other disease, one of the most important prerequisites is the ability to quantify miRNAs from a variety of samples with sufficient sensitivity and reproducibility. However, the function and molecular mechanism of miR-193b-3p and miR-376a-3p in CLL disease are yet unknown. In this study, miR-193b-3p and miR-376a-3p were selected from miRWalk database, as these two miRNAs are involved in CLL disease. Subsequently, the expression of miR-193b$3 p$ and miR-376a-3p was assessed by quantitative real-time PCR in 30 patients with CLL and 30 healthy individuals. The data revealed a reduced expression of both the miRNAs in patients with CLL compared to healthy individuals. According to our results, downregulation of miR-193b-3p and miR-376a-3p may have functional importance and be associated with poor prognosis of CLL. Changes in the expression of miR-193b-3p have been associated with various human cancers such as ALL, prostate cancer, and ovarian cancer [13-15]. Mets et al. suggested that the novel tumor-suppressor, miR-193b-3p, can cooperate with NOTCH1 in the suppression T-ALL, by targeting the MYB oncogene in T-ALL [13]. Rauhala et al (2010) reported that miR-193b expression was decreased with increased methylation in clinical prostate cancer when compared to benign prostatic hyperplasia. This miRNA is an epigenetically regulated putative tumor suppressor in prostate cancer [14]. Furthermore, it has been shown that down-regulation of miR-193b in ovarian cancer cell lines can play a role as a novel biomarker for ovarian cancer [15]. On the one hand, the deregulation of miR-193b probably affects cell growth in colon cancer through the TGF- $\beta$ and SMAD3 signaling pathways [26]. On the other hand, evidence has demonstrated that the expression of SMAD proteins plays an important role in CLL development [27]. Thus, miR-193b-3p may be useful as a biomarker for early diagnosis of different cancers.

Herr et al (2017) reported that miR-127 and miR-376a acted as strong tumor suppressors by in vivo targeting of COA1 and PDIA6 in a giant cell tumor of bone [16]. Meng et al (2015) demonstrated that miR-7, miR-16, miR-25, miR-93, miR-182, miR-376a, and miR429 show prognostic potential in serum of ovarian cancer patients [17]. Additionally, it has been suggested that down-regulation of miR-376a may contribute to the development of hepatocellular carcinoma by targeting $\mathrm{p} 85 \alpha$ [18]. It has been proposed that miR-376a 
regulates primordial follicle assembly by modulating the expression of proliferating cell nuclear antigen (Pcna) [28]. In CLL, Pcna levels are indicative of cell proliferation, clinical stage, and the lymphocyte doubling time [29]. Therefore, circulating miR-376a-3p can be used as a diagnostic and therapeutic biomarker. According to the molecular enrichment analysis, it can be assumed that $m i R-193 b-3 p$ and $m i R-376 a-3 p$ may have multiple target genes. Given that a miRNA has multiple targets, these different target genes may serve to raise important questions for future studies. Taken together, the findings of the current study showed the relevance of miR-193b-3p and miR-376a-3p to CLL. Consequently, in the future, miR-193b-3p and miR-376a-3p may be used as potential biomarkers for early diagnosis of patients with CLL. Understanding the complexity of miRNAs may open up a new vista to find biomarkers for clinical diagnosis of cancer and to monitor efficacy of various therapies. In conclusion, the present study suggests the role of miR-193b-3p and miR-376a-3p in CLL disease. Our data revealed that the expression levels of these miRNAs were downregulated in CLL. Thus, our results provide new insights regarding miRNAs, and miR-193b-3p and miR-376a-3p might serve as potential therapeutic targets or prevent disease progression. Although, it can be assumed from our results that miR$193 b-3 p$ and miR-376a-3p may have tumor-suppressing roles in CLL, more studies on larger scales are required to confirm this concept.

\section{Acknowledgements}

We thank all patients who participated in this study.

\section{Conflict of Interest}

The authors declare that they have no conflicts of interest.

\section{References}

[1] Gao C, Zhou C, Zhuang J, Liu L, Wei J, Liu C, Li H, Sun C. Identification of key candidate genes and miRNA-mRNA target pairs in chronic lymphocytic leukemia by integrated bioinformatics analysis. Mol Med Rep. 2019; 19(1):362-74.

[2] Bueso-Ramos CE, Ferrajoli A, Medeiros LJ, Keating MJ, Estrov Z. Aberrant morphology, proliferation, and apoptosis of B-cell chronic lymphocytic leukemia cells. Hematology. 2004; 9:279-86. 
[3] Chiorazzi N. Cell proliferation and death: forgotten features of chronic lymphocytic leukemia B cells. Best Pract Res Clin Haematol. 2007;20:399-413. 10.1016/j.beha.2007.03.007.

[4] DeSantis CE, Lin CC, Mariotto AB, Siegel RL, Stein KD, Kramer JL, Alteri R, Robbins AS and Jemal A. Cancer treatment and survivorship statistics. CA Cancer J Clin. 2014; 64:252-71.

[5] Sgambati M, Linet M, Devesa S. Chronic lymphocytic leukemia, epidemiological, familial, and genetic aspects. Chronic lymphocytic leukemias, Revised and Expanded, 2nd edn. In: Cheson B, (ed). Marcel Dekker, Inc: New York, NY, USA, 2001 pp 33-62.

[6] Mirzaei H, Fathullahzadeh S, Khanmohammadi R, Darijani M, Momeni F, Masoudifar $A$, et al. State of the art in microRNA as diagnostic and therapeutic biomarkers in chronic lymphocytic leukemia. J Cell Physiol. 2018; 233(2):888-900.

[7] Fernando TR, Rodriguez-Malave NI and Rao DS. MicroRNAs in B cell development and malignancy. J Hematol Oncol. 2012;5:7.

[8] Martini V, Frezzato F, Severin F, Raggi F, Trimarco V, Martinello L, Molfetta R, Visentin A, Facco M, Semenzato G, Paolini R. Abnormal regulation of BCR signalling by $\mathrm{c}-\mathrm{Cbl}$ in chronic lymphocytic leukaemia. Oncotarget. 2018;9:32219-31.

[9] Hershkovitz-Rokah O, Geva P, Salmon-Divon M, Shpilberg O, Liberman-Aronov S. Network analysis of microRNAs, genes and their regulation in diffuse and follicular B-cell lymphomas. Oncotarget. 2018; 9(8):28-79.

[10] Reddy KB. MicroRNA (miRNA) in cancer. Cancer Cell Int. 2015;15:38-42.

[11] Calin GA, Dumitru CD, Shimizu M, Bichi R, Zupo S, Noch E, et al. Frequent deletions and down-regulation of micro- RNA genes miR15 and miR16 at 13q14 in chronic lymphocytic leukemia. Proc Natl Acad Sci U S A. 2002;99(24):15524-9.

[12] Lawrie CH, Gal S, Dunlop HM, Pushkaran B, Liggins AP, Pulford K, et al. Detection of elevated levels of tumour-associated microRNAs in serum of patients with diffuse large B-cell lymphoma. Br J Haematol. 2008;141(5):672-5.

[13] Mets E, Van der Meulen J, Van Peer G, Boice M, Mestdagh P, Van de Walle I, et al. MicroRNA-193b-3p acts as a tumor suppressor by targeting the MYB oncogene in T-cell acute lymphoblastic leukemia. Leukemia. 2015;29(4):79-86.

[14] Rauhala HE, Jalava SE, Isotalo J, Bracken H, Lehmusvaara S, Tammela TL, et al. miR$193 \mathrm{~b}$ is an epigenetically regulated putative tumor suppressor in prostate cancer. Int J Cancer. 2010;127(6):1363-72.

[15] Li H, Xu Y, Qiu W, Zhao D, Zhang Y. Tissue miR-193b as a novel biomarker for patients with ovarian cancer. Med Sci Monit. 2015;21:3929. 
[16] Herr I, Sähr H, Zhao Z, Yin L, Omlor G, Lehner B, Fellenberg J. MiR-127 and miR-376a act as tumor suppressors by in vivo targeting of COA1 and PDIA6 in giant cell tumor of bone. Cancer Lett. 2017;409:49-55.

[17] Meng X, Joosse SA, Müller V, Trillsch F, Milde-Langosch K, Mahner S, et al. Diagnostic and prognostic potential of serum miR-7, miR-16, miR-25, miR-93, miR-182, miR-376a and miR-429 in ovarian cancer patients. Br J Cancer. 2015;113(9):1358.

[18] Zheng Y, Yin L, Chen H, Yang S, Pan C, Lu S, et al. miR-376a suppresses proliferation and induces apoptosis in hepatocellular carcinoma. FEBS Lett. 2012;586(16):2396403.

[19] Negrini M, Cutrona G, Bassi C, Fabris S, Zagatti B, Colombo M, et al. microRNAome expression in chronic lymphocytic leukemia: comparison with normal B-cell subsets and correlations with prognostic and clinical parameters. Clin Cancer Res. 2014;10:723.

[20] Schmittgen TD, Livak KJ. Analyzing real-time PCR data by the comparative C T method. Nat Protoc. 2008;3(6):1101.

[21] Xiao F, Zuo Z, Cai G, Kang S, Gao X, Li T. miRecords: an integrated resource for microRNA-target interactions. Nucleic Acids Res. 2008;37(suppl.1): D105-10.

[22] Hsu SD, Lin FM, Wu WY, Liang C, Huang WC, Chan WL, et al. miRTarBase: a database curates experimentally validated microRNA-target interactions. Nucleic Acids Res. 2010;39(suppl.1): D163-9.

[23] Fathullahzadeh S, Mirzaei H, Honardoost MA, Sahebkar A, Salehi M. Circulating microRNA-192 as a diagnostic biomarker in human chronic lymphocytic leukemia. Cancer Gene Ther. 2016;23(10):32-37.

[24] Moussay E, Wang K, Cho JH, van Moer K, Pierson S, Paggetti J, Nazarov PV, Palissot V, Hood LE, Berchem G, Galas DJ. MicroRNA as biomarkers and regulators in B-cell chronic lymphocytic leukemia. Proc Natl Acad Sci. 2011;108(16):6573-8.

[25] Filip AA, Grenda A, Popek S, Koczkodaj D, Michalak-Wojnowska M, Budzyński M, Wąsik-Szczepanek E, Zmorzyński S, Karczmarczyk A, Giannopoulos K. Expression of circulating miRNAs associated with lymphocyte differentiation and activation in CLL-another piece in the puzzle. Ann Hematol. 2017;96(1):33-50.

[26] Wu K, Zhao Z, Ma J, Chen J, Peng J, Yang S, He Y. Deregulation of miR-193b affects the growth of colon cancer cells via transforming growth factor- $\beta$ and regulation of the SMAD3 pathway. Oncol Lett. 2017;13(4):2557-62.

[27] Witkowska M, Majchrzak A, Cebula-Obrzut B, Wawrzyniak E, Robak T, Smolewski P. The distribution and potential prognostic value of SMAD protein expression in chronic lymphocytic leukemia. Tumor Biol. 2017;39(3):1010428317694551. 
[28] Zhang H, Jiang X, Zhang Y, Xu B, Hua J, Ma T, Zheng W, Sun R, Shen W, Cooke HJ, Hao Q. microRNA 376a regulates follicle assembly by targeting Pcna in fetal and neonatal mouse ovaries. Reproduction. 2014;148(1):43-54.

[29] Giglio AD, O'brien S, Ford RJ, Manning J, Saya H, Keating M, Johnston D, Chamone DF, Deisseroth AB. Proliferating cell nuclear antigen (PCNA) expression in chronic lymphocytic leukemia (CLL). Leukemia Lymphoma. 1993;10(4-5):265-71. 